\title{
Distribution of EGFR amplification, combined chromosome 7 gain and chromosome 10 loss, and TERT promoter mutation in brain tumors and their potential for the reclassification of IDHwt astrocytoma to glioblastoma
}

Stichel, Damian ; Ebrahimi, Azadeh ; Reuss, David ; Schrimpf, Daniel ; Ono, Takahiro ; Shirahata, Mitsuaki ; Reifenberger, Guido ; Weller, Michael ; Hänggi, Daniel ; Wick, Wolfgang ; Herold-Mende, Christel ; Westphal, Manfred ; Brandner, Sebastian ; Pfister, Stefan M ; Capper, David ; Sahm, Felix ; von Deimling, Andreas

\begin{abstract}
EGFR amplification (EGFRamp), the combination of gain of chromosome 7 and loss of chromosome $10(7+/ 10-)$, and TERT promoter mutation (pTERTmut) are alterations frequently observed in adult IDH-wild-type (IDHwt) glioblastoma (GBM). In the absence of endothelial proliferation and/or necrosis, these alterations currently are considered to serve as a surrogate for upgrading IDHwt diffuse or anaplastic astrocytoma to GBM. Here, we set out to determine the distribution of EGFRamp, 7+/10-, and pTERTmut by analyzing high-resolution copy-number profiles and next-generation sequencing data of primary brain tumors. In addition, we addressed the question whether combinations of partial gains on chromosome 7 and partial losses on chromosome 10 exhibited a diagnostic and prognostic value similar to that of complete $7+/ 10-$. Several such combinations proved relevant and were combined as the $7 / 10$ signature. Our results demonstrate that EGFRamp and the 7/10 signature are closely associated with IDHwt GBM. In contrast, pTERTmut is less specific for IDHwt GBM. We conclude that, in the absence of endothelial proliferation and/or necrosis, the detection of EGFRamp is a very strong surrogate marker for the diagnosis of GBM in IDHwt diffuse astrocytic tumors. The 7/10 signature is also a strong surrogate marker. However, care should be taken to exclude pleomorphic xanthoastrocytoma. pTERTmut is less restricted to this entity and needs companion analysis by other molecular markers to serve as a surrogate for diagnosing IDHwt GBM. A combination of any two of EGFRamp, the 7/10 signature and pTERTmut, is highly specific for IDHwt GBM and the combination of all three alterations is frequent and exclusively seen in IDHwt GBM.
\end{abstract}

DOI: https://doi.org/10.1007/s00401-018-1905-0

Posted at the Zurich Open Repository and Archive, University of Zurich

ZORA URL: https://doi.org/10.5167/uzh-153711

Journal Article

Accepted Version

Originally published at:

Stichel, Damian; Ebrahimi, Azadeh; Reuss, David; Schrimpf, Daniel; Ono, Takahiro; Shirahata, Mitsuaki; Reifenberger, Guido; Weller, Michael; Hänggi, Daniel; Wick, Wolfgang; Herold-Mende, Christel; Westphal, Manfred; Brandner, Sebastian; Pfister, Stefan M; Capper, David; Sahm, Felix; von Deimling, Andreas (2018). Distribution of EGFR amplification, combined chromosome 7 gain and chromosome 10 
loss, and TERT promoter mutation in brain tumors and their potential for the reclassification of IDHwt astrocytoma to glioblastoma. Acta Neuropathologica, 136(5):793-803.

DOI: https://doi.org/10.1007/s00401-018-1905-0 


\title{
Distribution of EGFR amplification, combined 7gain/10loss and TERT promoter mutation in brain tumors and their potential for the reclassification of IDHwt astrocytoma to glioblastoma
}

\author{
Damian Stichel ${ }^{1 *}$, Azadeh Ebrahimi ${ }^{1,2 *}$, David Reuss ${ }^{1,2}$, Daniel Schrimpf ${ }^{1,2}$, Takahiro Ono ${ }^{3}$, Mitsuaki \\ Shirahata $^{4}$, Guido Reifenberger ${ }^{5}$, Michael Weller ${ }^{6}$, Daniel Hänggi ${ }^{7}$, Wolfgang Wick ${ }^{8}$, Christel Herold- \\ Mende $^{9}$, Manfred Westphal ${ }^{10}$, Sebastian Brandner ${ }^{11}$, Stefan Pfister ${ }^{12}$, David Capper ${ }^{1,2,13}$, Felix Sahm ${ }^{1,2}$ \\ and Andreas von Deimling ${ }^{1,2}$,
}

${ }^{1}$ Clinical Cooperation Unit Neuropathology, German Cancer Consortium (DKTK), German Cancer Research Center (DKFZ) Heidelberg, Germany

${ }^{2}$ Department of Neuropathology, Institute of Pathology, Heidelberg University Hospital, Heidelberg, Germany

${ }^{3}$ Department of Neurosurgery, Akita University Graduate School of Medicine, Akita, Japan ${ }^{4}$ Department of Neuro-Oncology/Neurosurgery, Saitama Medical University International Medical Center, Hidaka, Japan

${ }^{5}$ Department of Neuropathology, Heinrich Heine University, Moorenstr. 5, 40225 Düsseldorf, Germany ${ }^{6}$ Department of Neurology, University Hospital and University of Zurich, Zurich, Switzerland ${ }^{7}$ Department of Neurosurgery, University Medical Center Mannheim, University of Heidelberg ${ }^{8}$ Department of Neurology, Heidelberg University Hospital, Heidelberg, and Clinical Cooperation Unit Neurooncology, German Cancer Consortium (DKTK), German Cancer Research Center (DKFZ) Heidelberg, Germany

${ }^{9}$ Department of Neurosurgery, Heidelberg University Hospital, Heidelberg, Germany ${ }^{10}$ Department of Neurosurgery, University Hamburg-Eppendorf, Martinistr. 52, 20251 Hamburg, Germany

${ }^{11}$ Division of Neuropathology, The National Hospital for Neurology and Neurosurgery, University College London NHS Foundation Trust, and †Department of Neurodegeneration, UCL Institute of Neurology, London, UK,

${ }^{12}$ Hopp Children's Cancer Center at the NCT Heidelberg (KiTZ), andDivision of Pediatric Neurooncology, German Cancer Consortium (DKTK), German Cancer Research Center (DKFZ), and Department of Pediatric Oncology, Hematology and Immunology, University Hospital Heidelberg, Heidelberg, Germany

${ }^{13}$ Department of Neuropathology, University Hospital Heidelberg, ,and German Cancer Consortium (DKTK), Partner Site Berlin, German Cancer Research Center (DKFZ) Heidelberg,and Charité Universitätsmedizin Berlin, corporate member of Freie Universität Berlin, Humboldt-Universität zu Berlin, and Berlin Institute of Health, Department of Neuropathology

${ }^{*}$ Tese authors contributed equally to this work 
Stichel et al., 2

Corresponding author

Andreas von Deimling

Department for Neuropathology and CCU Neuropathology

University of Heidelberg and DKFZ

Im Neuenheimer Feld 224

69120 Heidelberg

Fon: +49-6221-56 4650

Fax: +49-6221-56 4566

andreas.vondeimling@med.uni-heidelberg.de 
Stichel et al., 3

\section{Abstract}

EGFR amplification (EGFRamp), the combination of gain of chromosome 7 and loss of chromosome 10 (7+/10-) and TERT promoter mutations (pTERTmut) are alterations frequently observed in glioblastoma (GBM). In absence of endothelial proliferations and/or necrosis, these alterations currently are considered to serve as a surrogate for upgrading IDH wildtype (IDHwt) astrocytoma to GBM. Here we set out to determine the distribution of EGFRamp, 7+/10- and pTERTmut by analyzing intracranial tumors with high resolution copy number profiles (CNP) and sequencing data available. In addition, we addressed the question whether combinations of partial gains on 7 and losses on 10 exhibited a diagnostic and prognostic value similar to that of complete $7+/ 10-$. Several such combinations proved relevant and were combined and designated $7 / 10$ signature. Our results demonstrate that EGFRamp and 7/10 are closely associated with GBM. In contrast, pTERTmut is less specific for GBM. We conclude that in the absence of endothelial proliferations and/or necrosis the detection of EGFRamp is a very strong surrogate marker for the diagnosis of GBM in diffuse astrocytic tumors. The $7 / 10$ signature is also a strong surrogate marker. However, care should be taken to exclude pleomorphic xanthoastrocytoma. pTERTmut is less restricted to this entity and needs companion analysis by other molecular markers in order to serve as a surrogate for diagnosing GBM. A combination of any two of EGFRamp, 7/10 and pTERTmut is highly specific for GBM and the combination of all three alterations is frequent and exclusively seen in GBM.

\section{Keywords}

EGFR amplification, 7gain, 10loss, 7+/10-, , 7+/10q-, TERT, promoter, glioblastoma, astrocytoma, pleomorphic xanthoastrocytoma 
Stichel et al., 4

\section{Introduction}

The so called IDH wildtype (IDHwt) astrocytoma comprises different tumors with the majority exhibiting molecular lesions and survival characteristics of glioblastoma (GBM). A pressing question is whether EGFR amplification, the presence of a combined gain on chromosome 7 and loss on chromosome 10 and TERT promoter mutation can serve as surrogate marker for upgrading IDHwt astrocytoma, by definition lacking necrosis, to glioblastoma.

The combination of gains on chromosome 7 and losses on chromosome 10 are characteristic molecular alterations in glioblastoma (GBM). Recent studies have demonstrated that astrocytic tumors not fulfilling the morphological criteria for GBM but exhibiting typical molecular features of GBM exhibit a clinical course similar to that of morphologically unequivocal GBM [5,11,24,30,31]. Therefore, the presence of the combination of 7 gain and 10 loss is considered as molecular marker for GBM. Little is known about the relevance of assessing individual arms of chromosomes 7 and 10, i.e. does gain of either arm of 7 and loss of either arm of 10 suffice or is gain of both arms on 7 and loss of both arms on 10 required. Gain of chromosome 7 and loss of chromosome 10 in GBM haves been initially detected by cytogenetic analyses [25] [4]. The typical constellation found in GBM cell lines was trisomy of chromosomes 7 and monosomy of chromosome 10 most likely as a result of error in mitotic disjunction.

EGFR amplification (EGFRamp) in GBM has been initially observed by molecular and cytogenetic analyses $[18,33][3,9]$. Roughly half of the GBM exhibit a strong amplification of this gene mostly due to the presence of numerous double minutes, the extra chromosomal elements containing additional EGFR copies [28]

Telomerase reverse transcriptase (TERT) encodes the catalytic subunit of the telomerase complex. TERT promoter mutation ( $\mathrm{p} T E R T$ mut) initially has been detected in melanoma [12,13]. Subsequent investigations also revealed high frequencies of $\mathrm{p} T E R T$ mut in GBM and in oligodendroglioma.

$[1,14,15,19,21,32]_{2}=$ and their potential use for subgrouping of glioma and in a diagnostic setting $[2,7,8,22]$.

Recent technology development allows generating high resolution copy number profiles (CNP) of the human chromosomes in tumor tissue based on next generation sequencing or array data. We set out to address the distribution of EGFRamp, 7+/10- and pTERTmut in human brain tumors based on CNP, generated from DNA methylation array data sets and from next generation sequencing data.

Furthermore, we explored the prognostic association of these alterations in brain tumors diagnosed as IDHwt astrocytoma. 
Stichel et al., 5

\section{Material and Methods}

\section{Patient cohorts}

The present analyses are based on data from three cohorts available for analysis at the Department. of Neuropathology of the University Heidelberg. From all patients in the three cohorts $450 \mathrm{~K} / 850 \mathrm{~K}$ methylation array data are available. All patients have received a methylation based diagnosis as previously described [6].

Cohort 1 contains 2.417 patients for whom next generation panel sequencing with a panel including the TERT gene and its promoter has been performed. Cohort 1 was employed for determining the distribution of $\mathrm{p}$ TERTmut, EGFRamp and 7+/10- in human brain tumors. Only one tumor per patient was included in cohort 1 . Table 1 lists all tumors included in cohort 1 sorted by methylation based diagnosis.

Cohort 2 including 10.826 brain tumor patients with have been analyzed using the lllumina 450K or $850 \mathrm{~K}$ platforms is the basis of the distribution of EGFRamp and 7+/10- in human brain tumors. Only one tumor per patient was included in cohort 2. Supplementary table 1 lists all tumors included in cohort 2 sorted by methylation based diagnosis [6] and respective number of patients. Cohort 2 encompasses all patients from cohort 1. Details on methylation classes can be obtained from www.molecularneuropathology.org.

Cohort 3 contains 939 patients from cohort 2 with survival data available. In contrast to cohorts 1 and 2 the histological diagnosis according to WHO 2016 [20] including the diagnosis of IDHwt astrocytoma was basis for survival analysis. Cohort 3 does not include any patients diagnosed as glioblastoma. There is a bias in cohort 3 because survival data have been acquired for specific tumor entities in previous studies.

\section{Generation and scoring of CNPs and mutations}

Methylation data were generated using the Illumina $450 \mathrm{~K}$ or $850 \mathrm{~K} / \mathrm{EPIC}$ platforms as previously described [6].The copy number variation plots were generated from the same raw data using the 'conumee' R package in Bioconductor (http://www.bioconductor.org packages/release/bioc/html / conumee.html). Figure 1 shows representative CNPs. Automated assessment of copy-number changes was performed using the results from conumee after additional baseline correction. EGFRamp was called amplified if the respective probes exhibited an intensity higher than 0.6 on a $\log 2$-scale.

Panel sequencing was performed a previously reported [26]. pTERTmut was scored if 10 or more reads were detected with a minimum of $10 \%$ of the reads showing either of the two TERT promoter mutations mutation.

\section{Statistics}

All patient sets were retrospectively compiled. The size of the respective sets was determined by availability of data and not by a power calculation. OS times were analyzed by the Kaplan-Meier method and compared with a log-rank test. $P$ values less than 0.05 were considered significant. Software R version 3.4 and packages survival and party were employed for analysis 
Stichel et al., 6

\section{Results and Discussion}

\section{Rationale and procedure}

We aimed at contributing to three questions: First, what is the incidence of combined 7/10 alterations, EGFRamp and $\mathrm{p} T E R T$ mut in human brain tumors. Second, are these three alterations suitable surrogate markers for diagnosing GBM in the absence of necrosis and microvascular proliferation? and third, which variants of $7 / 10$ alterations might be employed. The first two questions have been addressed by analyzing a series of 2,417 tumors (cohort 1) with both TERT promoter status and complete copy number profiles being available (table 1). Question three was addressed by analyzing an extended set of 10,826 patients (cohort 2) with complete copy number profiles available (supplementary table 1) and analyzing a subset thereof including 939 (cohort 3 ) patients with overall survival data available.

For all series, both, a classical histopathological and a methylation based diagnosis was available. Current WHO GBM includes the H3-G34mutant GBM, while the H3-K27 GBM and the IDH mutant (IDHmut) GBM have been separated from the group of IDHwt GBM. For all questions addressing association of the markers interrogated with survival, we adhered to the current WHO definition of GBM (all analyses involving cohort 3). For determination of frequencies we used the methylation based diagnosis as this is highly standardized and, therefore, more suitable for this type of question.

\section{Defining $7 / 10$ status}

An open question is whether $7+/ 10$ - is prognostically relevant only if both arms each chromosome are affected or if losses of only one arm per chromosome suffice. Further, how much of each chromosomal arm needs to be affected to score either a gain or a loss?- This question is of special interest in light of many data being FISH analysis based and, therefore, not providing representative information-at all. Our approach is array platform based thereby covering the entire chromosomal arms. We selected two different thresholds with one being $50 \%$ and the other $80 \%$ of chromosomal representation being gained or lost for calling the respective alteration in cohort 1 . Supplementary table 1 provides an overview of $7+$ and 10- combinations using both thresholds. In the predominant GBM subgroup characterized by gain of entire 7 and loss of entire 10,1185 patients ( $75 \%$ of all GBM) scored positive with the $80 \%$ and 1265 patients ( $81 \%$ of all GBM) with the $50 \%$ threshold (supplementary table 1). We therefore went on using the $50 \%$ threshold for all subsequent analyses. Of 9 possible combinations exhibiting both, gain of at least one arm on 7 and loss of at least one arm on $10,7+/ 10$ - represents the most frequent $(1265 / 1598 ; 79 \%)$ constellation followed by $7+/ 10 q-$ $(87 / 1598 ; 5 \%)$, by $7 p+/ 10-(74 / 1598 ; 5 \%)$ and by $7 q+/ 10-(70 / 1598 ; 4 \%)$ (supplementary table 1$)$. Next we analyzed which variants of gains and losses were associated with unfavorable clinical outcome. To this end we analyzed the respective combinations in patients from cohort 3 .

Of the nine possibilities for 7 gain and 10loss combinations we encountered $7+/ 10-(n=97), 7 q+/ 10$ $(n=12), 7+/ 10 q-(n=9), 7 q+/ 10 q-(n=7), 7 p+/ 10-(n=3)$, both $7+/ 10 p-$ and $7 q+/ 10 p-(n=1)$, and both $7 p+/ 10 p$ and $7 p+/ 10 q-(n=0)$ in patients from cohort 3 . Survival analysis was performed of patients exhibiting the combinations $7+/ 10-(n=97), 7 q+/ 10-(n=12), 7+/ 10 q-(n=9), 7 q+/ 10 q-(n=7)$ and 
$7 p+/ 10-(n=3)$. Three combinations, 7+/10- , 7q+/10- , 7+/10q- associated with poor survival similar to that of patients with GBM (figure $2 \mathrm{a}$ ). We therefore defined all patients with $7+/ 10-, 7+/ 10 \mathrm{q}$ - and $7 q+/ 10$ - as carrying the prognostic $7 / 10$ signature. Noteworthy, methylation based classification identified a substantial number of patients in cohort 3 without a $7 / 10$ signature but with typical survival characteristics of GBM (figure 2b)demonstrating the power of this method.

\section{Distribution of EGFRamp, 7/10 signature and TERT in human brain tumors}

Comparison of the distribution of all three parameters was performed using cohort 2 although for the distribution of EGFRamp and 7/10 signature a higher resolution can be obtained from cohort 1.

Frequent pTERTmut was observed in 363/544 (67\%) GBM, in 95/120 (79\%) oligodendroglioma, in $12 / 17(71 \%)$ melanoma, in 19/42 (45\%) medulloblastoma methylation subclass SHH A and in 7/34 (21\%) pleomorphic xanthoastrocytoma (PXA). Numbers for pTERTmut in entities with low mutation frequencies or entities with only few tumors analyzed are given in the table 1. Of the three parameters, TERT exhibited highest sensitivity (67\%) but lowest specificity (89\%) for glioblastoma (table 2).

The $7 / 10$ signature was more specific for GBM being seen in 323/544 (59\%) GBM, in 9/140 (6\%) high grade $I D H$ mut astrocytoma including IDHmut GBM and in 5/54 (9\%) medulloblastoma of methylation subgroup 4 (table 1). Sensitivity was $59 \%$ and specificity was $98 \%$ (table 2 ).

EGFRamp was observed in 196/544 (36\%) GBM showing lowest sensitivity (36\%) but highest specificity (100\%) for GBM (table 2).

On the entire scale pTERTmut (562/2417) is more frequent than EGFRamp (199/2417) or 7/10 (361/2417). The combinations of pTERTmut - EGFRamp (28 cases), pTERTmut - 7/10 (146 cases) and EGFRamp - 7/10 (30 cases) were strongly associated with GBM and the triple combination of pTERTmut - 7+/10- - EGFRamp (124 cases) was exclusively seen in GBM (table 1). The sensitivity of any combination of double or triple positives was $58 \%$ and the specificity was $99 \%$ (table 2 ).

\section{Distribution of EGFRamp, 7/10 signature and pTERTmut in GBM methylation classes}

Subdivision of IDHwt GBM by methylation based classification results in 7 subgroups. These subgroups exhibit striking differences in the frequencies of the tree parameters interrogated. Tumors of the methylation class glioblastoma, IDHwt, H3.3 G34 mutant ( $n=17)$ did not exhibit pTERTmut or EGFRamp. The 7/10 signature was observed only in four H3.3 G34 mutant GBM. This finding is quite similar to that in H3.3 K27 mutant GBM and arguing for separating the H3.3 G34 mutant GBM from the IDHwt GBM, too. Tumors of the methylation class glioblastoma, IDHwt, subclass MYCN $(\mathrm{n}=22)$ exhibited pTERTmut and EGFRamp in less than quarter of all cases and the 7/10 signature only in a single tumor. Methylation class glioblastoma, IDHwt, subclass RTK I $(\mathrm{n}=71)$ presented with $\mathrm{pTERTmut}$ in 55 cases (77\%), 18 (25\%) times EGFRamp and 46 (65\%) times the $7 / 10$ signature. Methylation class glioblastoma, IDHwt, subclass RTK II ( $\mathrm{n}=203)$ constituting the most frequent GBM subgroup presented with pTERTmut in 166 cases (83\%), $128(63 \%)$ times EGFRamp and $160(79 \%)$ times the $7 / 10$ signature. The methylation class glioblastoma, IDHwt, subclass RTK III $(\mathrm{n}=23)$ predominantly encountered in young patients exhibited 11 times (48\%) pTERTmut, 8 times (35\%) EGFRamp and 3 times $(13 \%) 7 / 10$. The methylation class glioblastoma, IDHwt, subclass mesenchymal $(n=157)$ is frequent and presented with 123 (78\%) pTERTmut, 37 (24\%) EGFRamp and 109 (69\%) 7/10 
signatures. And finally tumors falling into the methylation class glioblastoma, IDHwt, subclass midline $(n=51)$ which is a yet poorly characterized group of tumors with morphology and survival characteristics comparable to that of GBM [24] exhibited pTERTmut in 4 (8\%) and EGFRamp or a $7 / 10$ signature in neither cases.

Clearly as morphological GBM comprises all these methylation subgroups, the sensitivity of EGFRamp, $7 / 10$ signature and pTERTmut based grading is compromised by the low or absent prevalence in some of these subgroups.

\section{Single use of one of the three parameters EGFRamp, 7/10 signature and $\mathrm{p} T E R T$ mut}

A single molecular marker for diagnosing GBM in absence of necrosis would be a major contribution to daily routine diagnostics. While EGFRamp, pTERTmut or $7 / 10$ signature are very good candidates, single use is not warranted. pTERTmut obviously needs accompanying analysis of $I D H 1$ and $I D H 2$ in order to exclude oligodendroglioma and astrocytoma. Also the rare adult medulloblastoma [23] and anaplastic meningioma $[10,27]$ as well as SFT/Hemangiopericytoma frequently carry $\mathrm{pTERTmut}$ and need to be distinguished by appropriate testing. The presence of $p T E R T$ mut in PXA [16] should be addressed by testing for the BRAFV600E mutation typical for the latter [29]. However, an overlap with the rare epithelioid glioblastoma cannot be ruled out [17]. EGFRamp has highest specificity and $I D H$ testing can separate the rare occurrences in $I D H$ mutant glioma. The $7 / 10$ signature also needs additional testing for $I D H$ to separate for anaplastic diffuse glioma and for BRAF for its occasional occurrence in PXA (table 1).

\section{Conclusions}

Our data supports the single use of the molecular genetic markers EGFRamp, pTERTmut or $7 / 10$ signature as strong markers for the reclassification of IDHwt astrocytoma to GBM pending additional molecular tests. The combination of a positive finding for any two of the three parameters is highly specific for and the combination of all three parameters is exclusively seen in GBM.

\section{Acknowledgements}

This work was supported by German Cancer Aid (70112371) to AvD. We thank Viktoria Zeller, Ulrike Lass, Antje Habel, Ulrike Vogel, Katja Brast, Kerstin Lindenberg and Jochen Meyer for excellent technical assistance. We also thank the Microarray unit of the Genomics and Proteomics Core Facility, German Cancer Research Center (DKFZ), for providing methylation services. 
Stichel et al., 9

\section{Figure legends}

\section{Table 1}

Classifier prediction, EGFR, 7/10 and TERT status in cohort 1. Full text for abbreviated methylation classes is provided in supplementary table 1. A characterization of methylation classes by Classifier prediction can be obtained from www.molecularneuropathology.org.

\section{Table 2}

Sensitivity and specificity of $\mathrm{p} T E R T$ mut, EGFRamp and $7 / 10$ signature as single parameters and in combination for 544 GBM in a series of 2417 brain tumors.

\section{Figure 1}

Representative CNP-plots: a) GBM with 7+/10- and EGFRamp; b) GBM with 7+/10q-; c) PXA with $7+/ 10-$

\section{Figure 2.}

a) OS in 939 patients (cohort 3) which have been diagnosed as IDHwt glioma, excluding glioblastoma, stratified for different combinations of alterations of chromosomes 7 and 10. Of all possible combinations with losses on chromosomes 7 and 10, only $7+/ 10-, 7+/ 10 q-, 7 p+10-, 7 q+/ 10 q-$ and $7 q+/ 10$ - were represented more than 3 times. Survival of glioma patients with $7+/ 10-, 7+/ 10 q$ - and $7 q+/ 10$ - was significantly worse than that of patients without these alterations.

b) OS in167 patients from cohort 3 which in addition have received the classifier diagnosis GBM. Methylation based classification identifies 52 additional patients without a 7/10 signature. Black graph is a reference series of additional 261 patients diagnosed as GBM by both, histology and methylation based classification.

\section{Supplementary table 1}

Distribution of EGFRamp and status of chromosomes 7 and 10 in 10.826 tumors. Chromosome 7 and 10 status is given for two different thresholds requiring loss $>50 \%$ or $>80 \%$ of the respective arms. Combinations not qualifying for any form of a combined 7 gain and 10 loss are indicated by print in grey. 


\section{References}

1 Arita H, Narita Y, Fukushima S et al. (2013) Upregulating mutations in the TERT promoter commonly occur in adult malignant gliomas and are strongly associated with total $1 p 19 q$ loss. Acta Neuropathol:

2 Bell RJ, Rube HT, Xavier-Magalhaes A, Costa BM, Mancini A, Song JS, Costello JF (2016) Understanding TERT Promoter Mutations: A Common Path to Immortality. Mol Cancer Res 14: 315-323

3 Bigner SH, Burger PC, Wong AJ, Werner MH, Hamilton SR, Muhlbaier LH, Vogelstein B, Bigner DD (1988) Gene amplification in malignant human gliomas: clinical and histopathologic aspects. J Neuropath Exp Neurol 47: 191-205

$4 \quad$ Bigner SH, Mark J, Burger PC, Mahaley SM, Bullard DEJ, Muhlbaier LH, Bigner DD (1988) Specific chromosomal abnormalities in malignant human gliomas. Cancer Res 48: 405-411

5 Cancer Genome Atlas Research N, Brat DJ, Verhaak RG et al. (2015) Comprehensive, Integrative Genomic Analysis of Diffuse Lower-Grade Gliomas. The New England journal of medicine 372: 2481-2498

6 Capper D, Jones DTW, Sill M et al. (2018) DNA methylation-based classification of central nervous system tumours. Nature 555: 469-474

7 Ceccarelli M, Barthel FP, Malta TM et al. (2016) Molecular Profiling Reveals Biologically Discrete Subsets and Pathways of Progression in Diffuse Glioma. Cell 164: 550-563

8 Eckel-Passow JE, Lachance DH, Molinaro AM et al. (2015) Glioma Groups Based on 1p/19q, IDH, and TERT Promoter Mutations in Tumors. The New England journal of medicine 372: 2499-2508

9 Ekstrand AJ, James CD, Cavenee WK, Seliger B, Petterson RF, Collins VP (1991) Genes for epidermal growth factor receptor, transforming growth factor $\alpha$, and epidermal growth factor and their expression in human gliomas in vivo. Cancer Res 51: 2164-2172

10 Goutagny S, Nault JC, Mallet M, Henin D, Rossi JZ, Kalamarides M (2014) High incidence of activating TERT promoter mutations in meningiomas undergoing malignant progression. Brain Pathol 24: 184-189

11 Hasselblatt M, Jaber M, Reuss D et al. (Epub ahead of print) Diffuse Astrocytoma, IDHWildtype: A Dissolving Diagnosis. J Neuropathol Exp Neurol:

12 Horn S, Figl A, Rachakonda PS et al. (2013) TERT promoter mutations in familial and sporadic melanoma. Science 339: 959-961

13 Huang FW, Hodis E, Xu MJ, Kryukov GV, Chin L, Garraway LA (2013) Highly recurrent TERT promoter mutations in human melanoma. Science 339: 957-959

14 Killela PJ, Reitman ZJ, Jiao Y et al. (2013) TERT promoter mutations occur frequently in gliomas and a subset of tumors derived from cells with low rates of self-renewal. Proc Natl Acad Sci U S A 110: 6021-6026

15 Koelsche C, Renner M, Hartmann W et al. (2014) TERT promoter hotspot mutations are recurrent in myxoid liposarcomas but rare in other soft tissue sarcoma entities. Journal of experimental \& clinical cancer research : CR 33: 33

16 Koelsche C, Sahm F, Capper D et al. (2013) Distribution of TERT promoter mutations in pediatric and adult tumors of the nervous system. Acta Neuropathol 126: 907-915

17 Korshunov A, Chavez L, Sharma T et al. (Epub ahead of print) Epithelioid glioblastomas stratify into established diagnostic subsets upon integrated molecular analysis. Brain Pathol:

18 Libermann TA, Nusbaum HR, Razon N et al. (1985) Amplification, enhanced expression and possible rearrangement of EGF receptor in primary human brain tumors of glial origin. Nature 313: 144-147

19 Liu X, Wu G, Shan Y, Hartmann C, von Deimling A, Xing M (2013) Highly prevalent TERT promoter mutations in bladder cancer and gliobastoma. Cell Cycle 12: 1637-1638 
Louis D, Ohgaki H, Wiestler O, Cavenee WK (2016) World Health Organization Classification of Tumours of the Central Nervous System. In: Bosman F, Jaffe E, Lakhani S, Ohgaki H (eds) World Health Organization Classification of Tumours Revised 4th edition edn. IARC, City

21 Nonoguchi N, Ohta T, Oh JE, Kim YH, Kleihues P, Ohgaki H (2013) TERT promoter mutations in primary and secondary glioblastomas. Acta Neuropathol:

22 Pekmezci M, Rice T, Molinaro AM et al. (2017) Adult infiltrating gliomas with WHO 2016 integrated diagnosis: additional prognostic roles of ATRX and TERT. Acta Neuropathol 133: 1001-1016

23 Remke M, Ramaswamy V, Peacock J et al. (2013) TERT promoter mutations are highly recurrent in SHH subgroup medulloblastoma. Acta Neuropathol 126: 917-929

24 Reuss DE, Kratz A, Sahm F et al. (2015) Adult IDH wild type astrocytomas biologically and clinically resolve into other tumor entities. Acta Neuropathol 130: 407-417

25 Rey JA, Bello MJ, de Campos JM, Kusak E, Ramos C, Benitez J (1987) Chromosomal patterns in human malignant astrocytoma. Cancer Genet Cytogenet 29: 201-221

26 Sahm F, Schrimpf D, Jones DT et al. (2016) Next-generation sequencing in routine brain tumor diagnostics enables an integrated diagnosis and identifies actionable targets. Acta Neuropathol 131: 903-910

27 Sahm F, Schrimpf D, Olar A et al. (2015) TERT Promoter Mutations and Risk of Recurrence in Meningioma. J Natl Cancer Inst 108: djv377

28 Sauter G, Maeda T, Waldman FM, Davis RL, Feuerstein BG (1996) Patterns of epidermal growth factor receptor amplification in malignant gliomas. American Journal of Pathology 148: 1047-1053

29 Schindler G, Capper D, Meyer J et al. (2011) Analysis of BRAF V600E mutation in 1320 nervous system tumors reveals high mutation frequencies in pleomorphic xanthoastrocytoma and ganglioglioma. Acta Neuropathol 121: 397-405

30 Sun Y, Zhang W, Chen D et al. (2014) A glioma classification scheme based on coexpression modules of EGFR and PDGFRA. Proc Natl Acad Sci U S A 111: 3538-3543

31 Suzuki H, Aoki K, Chiba K et al. (2015) Mutational landscape and clonal architecture in grade II and III gliomas. Nat Genet 47: 458-468

32 Vinagre J, Almeida A, Populo $\mathrm{H}$ et al. (2013) Frequency of TERT promoter mutations in human cancers. Nature communications 4: 1-6

33 Wong AJ, Bigner SH, Bigner DD, Kinzler KW, Hamilton SR, Vogelstein B (1987) Increased expression of the epidermal growth factor receptor gene in malignant gliomas is invariably associated with gene amplification. Proc Natl Acad Sci USA 84: 6899-6903 
Stichel et al., 12

Table 1

\begin{tabular}{|c|c|c|c|c|c|c|c|c|c|c|c|c|}
\hline $\begin{array}{l}\text { Methylation class } \\
\text { abbreviation }\end{array}$ & $\begin{array}{c}n \\
\text { total }\end{array}$ & $\underset{T E R T}{\mathrm{n}}$ & $\stackrel{\mathrm{n}}{E G F R}$ & $\underset{7 / 10}{n}$ & $\begin{array}{c}\text { triple } \\
\text { wt }\end{array}$ & $\begin{array}{c}\text { single } \\
7 / 10\end{array}$ & $\begin{array}{l}\text { single } \\
\text { EGFR }\end{array}$ & $\begin{array}{l}\text { single } \\
\text { TERT }\end{array}$ & $\begin{array}{c}\text { double } \\
7 / 10+\text { EGFR }\end{array}$ & $\begin{array}{c}\text { double } \\
7 / 10+\text { TERT }\end{array}$ & $\begin{array}{c}\text { double } \\
\text { EGFR + TERT }\end{array}$ & $\begin{array}{l}\text { triple } \\
7 / 10+\text { EGFR + TERT }\end{array}$ \\
\hline PXA & 34 & 7 & 0 & 2 & 26 & 1 & 0 & 6 & 0 & 1 & 0 & 0 \\
\hline EFT_CIC & 3 & 0 & 0 & 0 & 3 & 0 & 0 & 0 & 0 & 0 & 0 & 0 \\
\hline HGNET_BCOR & 9 & 0 & 0 & 0 & 9 & 0 & 0 & 0 & 0 & 0 & 0 & 0 \\
\hline HGNET_MN1 & 6 & 0 & 0 & 0 & 6 & 0 & 0 & 0 & 0 & 0 & 0 & 0 \\
\hline CNS_NB_FOXXR2 & 1 & 0 & 0 & 0 & 1 & 0 & 0 & 0 & 0 & 0 & 0 & 0 \\
\hline EWS & 5 & 1 & 0 & 0 & 4 & 0 & 0 & 1 & 0 & 0 & 0 & 0 \\
\hline O_IDH & 120 & 95 & 0 & 1 & 25 & 0 & 0 & 94 & 0 & 1 & 0 & 0 \\
\hline A_IDH & 154 & 5 & 0 & 1 & 148 & 1 & 0 & 5 & 0 & 0 & 0 & 0 \\
\hline A_IDH_HG & 140 & 11 & 1 & 9 & 121 & 7 & 1 & 9 & 0 & 2 & 0 & 0 \\
\hline ANA_PA & 75 & 2 & 0 & 1 & 72 & 1 & 0 & 2 & 0 & 0 & 0 & 0 \\
\hline ATRT̄_MYC & 4 & 0 & 0 & 0 & 4 & 0 & 0 & 0 & 0 & 0 & 0 & 0 \\
\hline ATRT_SHH & 7 & 0 & 0 & 0 & 7 & 0 & 0 & 0 & 0 & 0 & 0 & 0 \\
\hline ATRT_TYR & 6 & 0 & 0 & 0 & 6 & 0 & 0 & 0 & 0 & 0 & 0 & 0 \\
\hline $\mathrm{CN}$ & 18 & 0 & 0 & 0 & 18 & 0 & 0 & 0 & 0 & 0 & 0 & 0 \\
\hline LIPN & 2 & 0 & 0 & 0 & 2 & 0 & 0 & 0 & 0 & 0 & 0 & 0 \\
\hline CHGL & 1 & 0 & 0 & 0 & 1 & 0 & 0 & 0 & 0 & 0 & 0 & 0 \\
\hline CHORDM & 4 & 1 & 1 & 2 & 2 & 0 & 0 & 0 & 1 & 1 & 0 & 0 \\
\hline CPH_ADM & 2 & 0 & 0 & 0 & 2 & 0 & 0 & 0 & 0 & 0 & 0 & 0 \\
\hline CPH_PAP & 4 & 0 & 0 & 0 & 4 & 0 & 0 & 0 & 0 & 0 & 0 & 0 \\
\hline DLGN̄T & 9 & 0 & 0 & 0 & 9 & 0 & 0 & 0 & 0 & 0 & 0 & 0 \\
\hline DMG_K27 & 63 & 0 & 0 & 0 & 63 & 0 & 0 & 0 & 0 & 0 & 0 & 0 \\
\hline ETMR & 25 & 0 & 0 & 0 & 25 & 0 & 0 & 0 & 0 & 0 & 0 & 0 \\
\hline EPN_RELA & 14 & 0 & 0 & 0 & 14 & 0 & 0 & 0 & 0 & 0 & 0 & 0 \\
\hline EPN_YAP & 1 & 0 & 0 & 0 & 1 & 0 & 0 & 0 & 0 & 0 & 0 & 0 \\
\hline EPN_MPE & 15 & 0 & 0 & 1 & 14 & 1 & 0 & 0 & 0 & 0 & 0 & 0 \\
\hline EPN_PF_A & 34 & 0 & 0 & 2 & 32 & 2 & 0 & 0 & 0 & 0 & 0 & 0 \\
\hline EPN_PF_B & 6 & 1 & 0 & 0 & 5 & 0 & 0 & 1 & 0 & 0 & 0 & 0 \\
\hline EPN_SPINE & 4 & 0 & 0 & 0 & 4 & 0 & 0 & 0 & 0 & 0 & 0 & 0 \\
\hline ENB_A & 3 & 0 & 0 & 1 & 2 & 1 & 0 & 0 & 0 & 0 & 0 & 0 \\
\hline ENB_B & 2 & 0 & 0 & 0 & 2 & 0 & 0 & 0 & 0 & 0 & 0 & 0 \\
\hline GBM_G34 & 17 & 0 & 0 & 4 & 13 & 4 & 0 & 0 & 0 & 0 & 0 & 0 \\
\hline GBM_MYCN & 22 & 4 & 5 & 1 & 14 & 0 & 4 & 2 & 0 & 1 & 1 & 0 \\
\hline GBM_RTK_I & 71 & 55 & 18 & 46 & 4 & 8 & 2 & 13 & 2 & 28 & 6 & 8 \\
\hline GBM_RTK_II & 203 & 166 & 128 & 160 & 6 & 7 & 4 & 21 & 20 & 41 & 12 & 92 \\
\hline GBM_RTK_III & 23 & 11 & 8 & 3 & 7 & 0 & 4 & 6 & 1 & 2 & 3 & 0 \\
\hline GBM_MES & 157 & 123 & 37 & 109 & 12 & 15 & 1 & 29 & 6 & 64 & 6 & 24 \\
\hline GBM_MID & 51 & 4 & 0 & 0 & 47 & 0 & 0 & 4 & 0 & 0 & 0 & 0 \\
\hline $\mathrm{HMB}$ & 4 & 0 & 0 & 0 & 4 & 0 & 0 & 0 & 0 & 0 & 0 & 0 \\
\hline IHG & 7 & 1 & 0 & 0 & 6 & 0 & 0 & 1 & 0 & 0 & 0 & 0 \\
\hline LGG_MYB & 14 & 0 & 0 & 0 & 14 & 0 & 0 & 0 & 0 & 0 & 0 & 0 \\
\hline LGG_DIG_DIA & 3 & 0 & 0 & 0 & 3 & 0 & 0 & 0 & 0 & 0 & 0 & 0 \\
\hline LGG_DNT & 19 & 0 & 0 & 0 & 19 & 0 & 0 & 0 & 0 & 0 & 0 & 0 \\
\hline LGG_GG & 12 & 3 & 0 & 2 & 9 & 0 & 0 & 1 & 0 & 2 & 0 & 0 \\
\hline LGG_RGNT & 8 & 0 & 0 & 0 & 8 & 0 & 0 & 0 & 0 & 0 & 0 & 0 \\
\hline LGG_PA_GG_ST & 30 & 1 & 0 & 1 & 29 & 0 & 0 & 0 & 0 & 1 & 0 & 0 \\
\hline LGG_PA_MID & 34 & 0 & 0 & 0 & 34 & 0 & 0 & 0 & 0 & 0 & 0 & 0 \\
\hline LGG_PA_PF & 78 & 0 & 0 & 0 & 78 & 0 & 0 & 0 & 0 & 0 & 0 & 0 \\
\hline LGG_SEGA & 13 & 0 & 0 & 0 & 13 & 0 & 0 & 0 & 0 & 0 & 0 & 0 \\
\hline LYMP̄HO & 13 & 0 & 0 & 0 & 13 & 0 & 0 & 0 & 0 & 0 & 0 & 0 \\
\hline MB_WNT & 34 & 2 & 0 & 0 & 32 & 0 & 0 & 2 & 0 & 0 & 0 & 0 \\
\hline MB_SHH_CHL_AD & 42 & 19 & 0 & 0 & 23 & 0 & 0 & 19 & 0 & 0 & 0 & 0 \\
\hline MB_SHH_INF & 47 & 1 & 0 & 0 & 46 & 0 & 0 & 1 & 0 & 0 & 0 & 0 \\
\hline MB_G3 & 33 & 0 & 0 & 1 & 32 & 1 & 0 & 0 & 0 & 0 & 0 & 0 \\
\hline MB_G4 & 54 & 4 & 0 & 5 & 45 & 5 & 0 & 4 & 0 & 0 & 0 & 0 \\
\hline MELCYYT & 17 & 0 & 0 & 0 & 17 & 0 & 0 & 0 & 0 & 0 & 0 & 0 \\
\hline MELAN & 17 & 12 & 0 & 1 & 5 & 0 & 0 & 11 & 0 & 1 & 0 & 0 \\
\hline SCHW_MEL & 8 & 1 & 0 & 1 & 6 & 1 & 0 & 1 & 0 & 0 & 0 & 0 \\
\hline MNG & 476 & 22 & 0 & 2 & 453 & 1 & 0 & 21 & 0 & 1 & 0 & 0 \\
\hline PTPR_A & 4 & 0 & 0 & 0 & 4 & 0 & 0 & 0 & 0 & 0 & 0 & 0 \\
\hline PTPR_B & 14 & 0 & 0 & 0 & 14 & 0 & 0 & 0 & 0 & 0 & 0 & 0 \\
\hline PGG_nC & 8 & 0 & 0 & 0 & 8 & 0 & 0 & 0 & 0 & 0 & 0 & 0 \\
\hline PIN_T̄_PPT & 12 & 0 & 0 & 0 & 12 & 0 & 0 & 0 & 0 & 0 & 0 & 0 \\
\hline PIN_T_PB_A & 3 & 0 & 0 & 1 & 2 & 1 & 0 & 0 & 0 & 0 & 0 & 0 \\
\hline PIN_T_PB_B & 7 & 0 & 0 & 1 & 6 & 1 & 0 & 0 & 0 & 0 & 0 & 0 \\
\hline PITÜI- & 5 & 2 & 0 & 0 & 3 & 0 & 0 & 2 & 0 & 0 & 0 & 0 \\
\hline PITAD_STH_DNS_B & 1 & 0 & 0 & 0 & 1 & 0 & 0 & 0 & 0 & 0 & 0 & 0 \\
\hline PLEX_AD & 5 & 0 & 0 & 1 & 4 & 1 & 0 & 0 & 0 & 0 & 0 & 0 \\
\hline PLEX_PED_A & 6 & 0 & 0 & 2 & 4 & 2 & 0 & 0 & 0 & 0 & 0 & 0 \\
\hline PLEX_PED_B & 28 & 3 & 1 & 0 & 24 & 0 & 1 & 3 & 0 & 0 & 0 & 0 \\
\hline SCHW & 18 & 0 & 0 & 0 & 18 & 0 & 0 & 0 & 0 & 0 & 0 & 0 \\
\hline SFT_HMPC & 16 & 4 & 0 & 0 & 12 & 0 & 0 & 4 & 0 & 0 & 0 & 0 \\
\hline SUBEEPN_PF & 2 & 1 & 0 & 0 & 1 & 0 & 0 & 1 & 0 & 0 & 0 & 0 \\
\hline SUBEPN SPINE & 4 & 0 & 0 & 0 & 4 & 0 & 0 & 0 & 0 & 0 & 0 & 0 \\
\hline SUBEPN_ST & 6 & 0 & 0 & 0 & 6 & 0 & 0 & 0 & 0 & 0 & 0 & 0 \\
\hline sum & 2417 & 562 & 199 & 361 & 1747 & 61 & 17 & 264 & 30 & 146 & 28 & 124 \\
\hline
\end{tabular}


Stichel et al., 13

Table 2

\begin{tabular}{|c|c|c|c|c|c|c|c|c|}
\hline $\mathbf{n}$ & $\mathbf{n}$ & $\mathbf{n}$ & $\mathbf{n}$ & double & double & double & triple & any double \\
\hline total & TERT & EGFR & $7 / 10$ & $7 / 10+E G F R$ & $7 / 10+T E R T$ & $E G F R+T E R T$ & $7 / 10+E G F R+T E R T$ & or triple \\
\hline true positive & 363 & 196 & 323 & 29 & 136 & 28 & 124 & 317 \\
\hline true negative & 1674 & 1870 & 1835 & 1872 & 1863 & 1873 & 1873 & 1862 \\
\hline false positive & 199 & 3 & 38 & 1 & 10 & 0 & 0 & 11 \\
\hline false negative & 181 & 348 & 221 & 515 & 408 & 516 & 420 & 227 \\
\hline sensitivity & $66,7 \%$ & $36,0 \%$ & $59,4 \%$ & $5,3 \%$ & $25,0 \%$ & $5,1 \%$ & $22,8 \%$ & $58,3 \%$ \\
\hline specificity & $89,4 \%$ & $99,8 \%$ & $98,0 \%$ & $99,9 \%$ & $99,5 \%$ & $100,0 \%$ & $100,0 \%$ & $99,4 \%$ \\
\hline
\end{tabular}


Stichel et al., 14

Figure 1
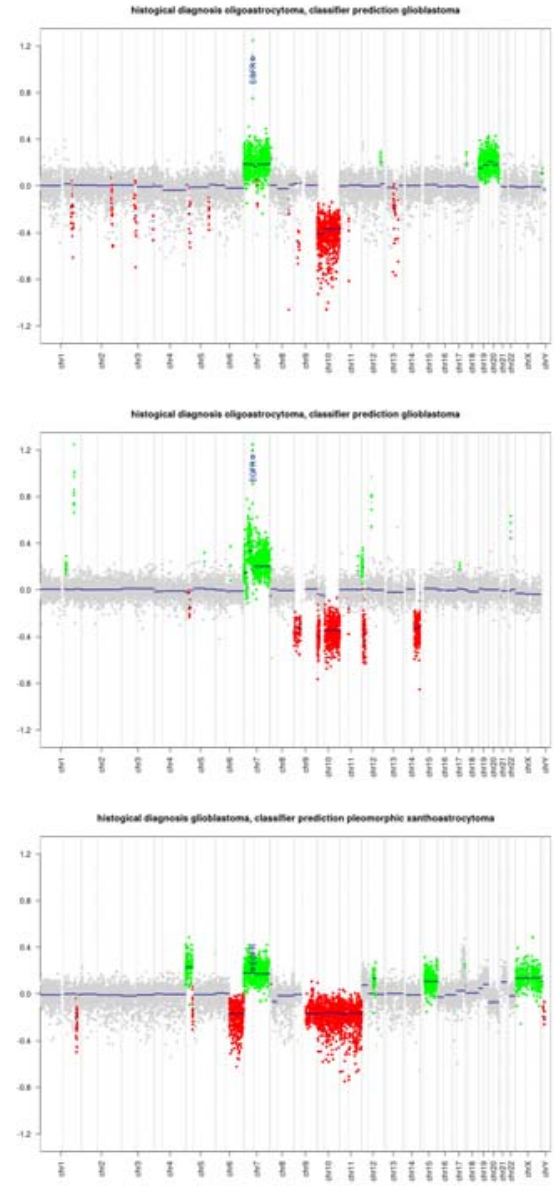
Stichel et al., 15

Figure 2
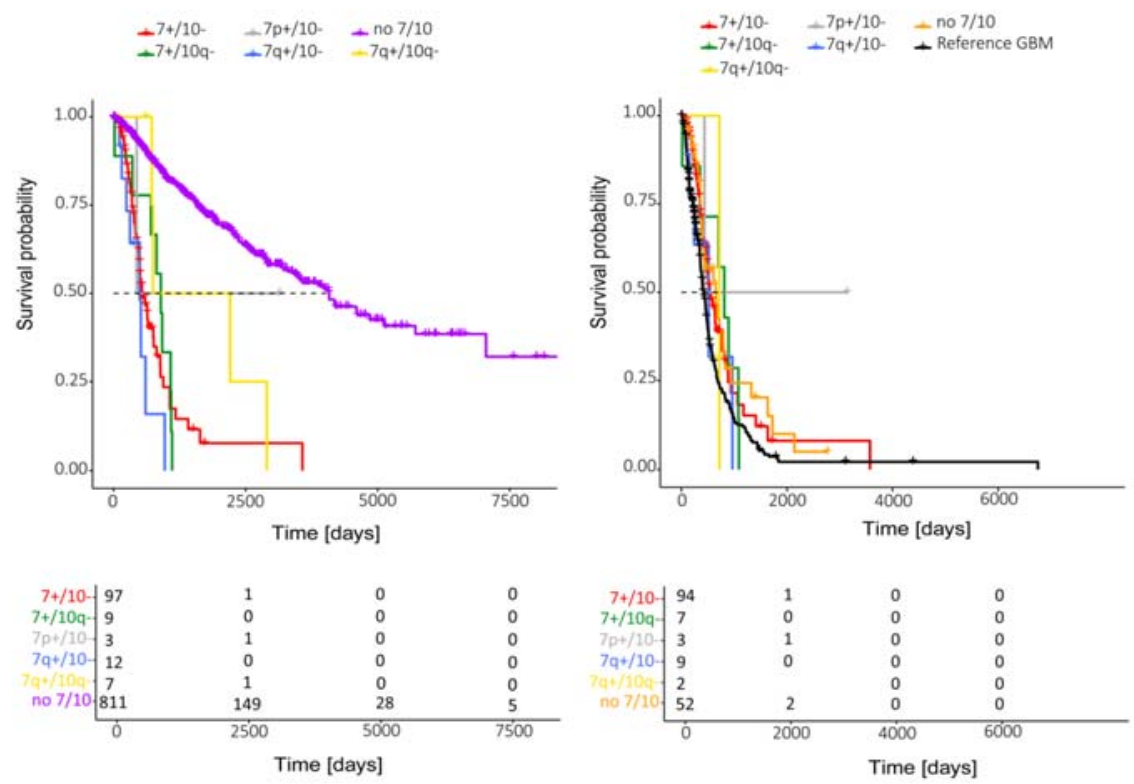


\section{methylation class}

1

2

3

4

5

6

7

8

9

Methylation class (anaplastic) pleomorphic xanthoastrocytoma

Methylation class CNS Ewing sarcoma family tumor with CIC alteration

Methylation class CNS high grade neuroepithelial tumor with BCOR alteration

Methylation class CNS high grade neuroepithelial tumor with MN1 alteration

Methylation class CNS neuroblastoma with FOXR2 activation

Methylation class Ewing sarcoma

Methylation class IDH glioma, subclass $1 \mathrm{p} / 19 \mathrm{q}$ codeleted oligodendroglioma

Methylation class IDH glioma, subclass astrocytoma

Methylation class IDH glioma, subclass high grade astrocytoma

Methylation class anaplastic pilocytic astrocytoma

Methylation class atypical teratoid/rhabdoid tumor, subclass MYC

Methylation class atypical teratoid/rhabdoid tumor, subclass $\mathrm{SHH}$

Methylation class atypical teratoid/rhabdoid tumor, subclass TYR

Methylation class central neurocytoma

Methylation class cerebellar liponeurocytoma

Methylation class chordoid glioma of the third ventricle

Methylation class chordoma

Methylation class craniopharyngioma, adamantinomatous

Methylation class craniopharyngioma, papillary

Methylation class diffuse leptomeningeal glioneuronal tumor

Methylation class diffuse midline glioma H3 K27M mutant

Methylation class embryonal tumor with multilayered rosettes

Methylation class ependymoma, RELA fusion

Methylation class ependymoma, YAP fusion 
Methylation class ependymoma, myxopapillary

Methylation class ependymoma, posterior fossa group A Methylation class ependymoma, posterior fossa group B Methylation class ependymoma, spinal Methylation class esthesioneuroblastoma, subclass A Methylation class esthesioneuroblastoma, subclass B Methylation class glioblastoma, IDH wildtype, H3.3 G34 mutant Methylation class glioblastoma, IDH wildtype, subclass MYCN Methylation class glioblastoma, IDH wildtype, subclass RTK I Methylation class glioblastoma, IDH wildtype, subclass RTK II Methylation class glioblastoma, IDH wildtype, subclass RTK III Methylation class glioblastoma, IDH wildtype, subclass mesenchymal Methylation class glioblastoma, IDH wildtype, subclass midline Methylation class hemangioblastoma Methylation class infantile hemispheric glioma Methylation class low grade glioma, MYB/MYBL1

thylation class low grade glioma, desmoplastic infantile astrocytoma / ganglioglio Methylation class low grade glioma, dysembryoplastic neuroepithelial tumor Methylation class low grade glioma, ganglioglioma Methylation class low grade glioma, rosette forming glioneuronal tumor ion class low grade glioma, subclass hemispheric pilocytic astrocytoma and gang Methylation class low grade glioma, subclass midline pilocytic astrocytoma Uethylation class low grade glioma, subclass posterior fossa pilocytic astrocytom: Methylation class low grade glioma, subependymal giant cell astrocytoma Methylation class lymphoma 
亏HH_Methylation class medulloblastoma, subclass $\mathrm{SHH}$ A (children and adultCH Methylation class medulloblastoma, subclass SHH B (infant) Methylation class medulloblastoma, subclass group 3 Methylation class medulloblastoma, subclass group 4 Methylation class melanocytoma Methylation class melanoma Methylation class melanotic schwannoma Methylation class meningioma Methylation class papillary tumor of the pineal region group $A$ Methylation class papillary tumor of the pineal region group $B$ Methylation class paraganglioma, spinal non-CIMP Methylation class pineal parenchymal tumor Methylation class pineoblastoma group A / intracranial retinoblastoma Methylation class pineoblastoma group B Methylation class pituicytoma / granular cell tumor / spindle cell oncocytoma Methylation class pituitary adenoma, ACTH Methylation class pituitary adenoma, $\mathrm{FSH} / \mathrm{LH}$ Methylation class pituitary adenoma, STH densely granulated, group A Methylation class pituitary adenoma, STH densely granulated, group B Methylation class pituitary adenoma, STH sparsely granulated Methylation class pituitary adenoma, TSH PITAD_Methylation class pituitary adenoma, prolactinPRL Methylation class plasmacytoma Methylation class plexus tumor, subclass adult 
75

76

78

79

80

81

82

Methylation class plexus tumor, subclass pediatric $\mathrm{A}$

Methylation class plexus tumor, subclass pediatric $\mathrm{B}$

Methylation class schwannoma

Methylation class solitary fibrous tumor / hemangiopericytoma

Methylation class subependymoma, posterior fossa

Methylation class subependymoma, spinal

Methylation class subependymoma, supratentorial 


\begin{tabular}{|c|c|c|c|c|c|}
\hline abbrev. & $\mathrm{n}$ & $E_{G F R_{\text {amp }}}$ & $\mathrm{EGFR}_{\mathrm{wt}}$ & EGFR $_{\text {amp }} \%$ & threshold \% \\
\hline PXA & 199 & 0 & 199 & 0.0 & $\begin{array}{l}50 \\
80\end{array}$ \\
\hline EFT_CIC & 85 & 0 & 85 & 0.0 & $\begin{array}{l}50 \\
80\end{array}$ \\
\hline HGNET_BCOR & 62 & 0 & 62 & 0.0 & $\begin{array}{l}50 \\
80\end{array}$ \\
\hline HGNET_MN1 & 58 & 0 & 58 & 0.0 & $\begin{array}{l}50 \\
80\end{array}$ \\
\hline CNS_NB_FOXR2 & 56 & 0 & 56 & 0.0 & $\begin{array}{l}50 \\
80\end{array}$ \\
\hline EWS & 121 & 0 & 121 & 0.0 & $\begin{array}{l}50 \\
80\end{array}$ \\
\hline O_IDH & 528 & 1 & 527 & 0.2 & $\begin{array}{l}50 \\
80\end{array}$ \\
\hline A_IDH & 640 & 0 & 640 & 0.0 & $\begin{array}{l}50 \\
80\end{array}$ \\
\hline A_IDH_HG & 351 & 4 & 347 & 1.1 & $\begin{array}{l}50 \\
80\end{array}$ \\
\hline ANA_PA & 112 & 0 & 112 & 0.0 & $\begin{array}{l}50 \\
80\end{array}$ \\
\hline ATRT_MYC & 81 & 0 & 81 & 0.0 & $\begin{array}{l}50 \\
80\end{array}$ \\
\hline ATRT_SHH & 109 & 0 & 109 & 0.0 & $\begin{array}{l}50 \\
80\end{array}$ \\
\hline ATRT_TYR & 61 & 0 & 61 & 0.0 & $\begin{array}{l}50 \\
80\end{array}$ \\
\hline $\mathrm{CN}$ & 30 & 0 & 30 & 0.0 & $\begin{array}{l}50 \\
80\end{array}$ \\
\hline LIPN & 16 & 0 & 16 & 0.0 & $\begin{array}{l}50 \\
80\end{array}$ \\
\hline CHGL & 17 & 0 & 17 & 0.0 & $\begin{array}{l}50 \\
80\end{array}$ \\
\hline CHORDM & 81 & 1 & 80 & 1.2 & $\begin{array}{l}50 \\
80\end{array}$ \\
\hline CPH_ADM & 41 & 0 & 41 & 0.0 & $\begin{array}{l}50 \\
80\end{array}$ \\
\hline CPH_PAP & 49 & 0 & 49 & 0.0 & $\begin{array}{l}50 \\
80\end{array}$ \\
\hline DLGNT & 29 & 0 & 29 & 0.0 & $\begin{array}{l}50 \\
80\end{array}$ \\
\hline DMG_K27 & 292 & 4 & 288 & 1.4 & $\begin{array}{l}50 \\
80\end{array}$ \\
\hline ETMR & 106 & 0 & 106 & 0.0 & $\begin{array}{l}50 \\
80\end{array}$ \\
\hline EPN_RELA & 161 & 0 & 161 & 0.0 & $\begin{array}{l}50 \\
80\end{array}$ \\
\hline EPN_YAP & 15 & 0 & 15 & 0.0 & $\begin{array}{l}50 \\
80\end{array}$ \\
\hline
\end{tabular}




\begin{tabular}{|c|c|c|c|c|c|}
\hline EPN_MPE & 76 & 1 & 75 & 1.3 & $\begin{array}{l}50 \\
80\end{array}$ \\
\hline EPN_PF_A & 367 & 0 & 367 & 0.0 & $\begin{array}{l}50 \\
80\end{array}$ \\
\hline EPN_PF_B & 95 & 0 & 95 & 0.0 & $\begin{array}{l}50 \\
80\end{array}$ \\
\hline EPN_SPINE & 59 & 0 & 59 & 0.0 & $\begin{array}{l}50 \\
80\end{array}$ \\
\hline ENB_A & 23 & 0 & 23 & 0.0 & $\begin{array}{l}50 \\
80\end{array}$ \\
\hline ENB_B & 22 & 0 & 22 & 0.0 & $\begin{array}{l}50 \\
80\end{array}$ \\
\hline GBM_G34 & 105 & 4 & 101 & 3.8 & $\begin{array}{l}50 \\
80\end{array}$ \\
\hline GBM_MYCN & 47 & 12 & 35 & 25.5 & $\begin{array}{l}50 \\
80\end{array}$ \\
\hline GBM_RTK_I & 350 & 73 & 277 & 20.9 & $\begin{array}{l}50 \\
80\end{array}$ \\
\hline GBM_RTK_II & 873 & 599 & 274 & 68.6 & $\begin{array}{l}50 \\
80\end{array}$ \\
\hline GBM_RTK_III & 42 & 13 & 29 & 31.0 & $\begin{array}{l}50 \\
80\end{array}$ \\
\hline GBM_MES & 705 & 197 & 508 & 27.9 & $\begin{array}{l}50 \\
80\end{array}$ \\
\hline GBM_MID & 125 & 0 & 125 & 0.0 & $\begin{array}{l}50 \\
80\end{array}$ \\
\hline $\mathrm{HMB}$ & 57 & 0 & 57 & 0.0 & $\begin{array}{l}50 \\
80\end{array}$ \\
\hline IHG & 37 & 0 & 37 & 0.0 & $\begin{array}{l}50 \\
80\end{array}$ \\
\hline LGG_MYB & 88 & 0 & 88 & 0.0 & $\begin{array}{l}50 \\
80\end{array}$ \\
\hline LGG_DIG_DIA & 18 & 0 & 18 & 0.0 & $\begin{array}{l}50 \\
80\end{array}$ \\
\hline LGG_DNT & 136 & 0 & 136 & 0.0 & $\begin{array}{l}50 \\
80\end{array}$ \\
\hline LGG_GG & 75 & 0 & 75 & 0.0 & $\begin{array}{l}50 \\
80\end{array}$ \\
\hline LGG_RGNT & 26 & 0 & 26 & 0.0 & $\begin{array}{l}50 \\
80\end{array}$ \\
\hline LGG_PA_GG_ST & 120 & 0 & 120 & 0.0 & $\begin{array}{l}50 \\
80\end{array}$ \\
\hline LGG_PA_MID & 115 & 0 & 115 & 0.0 & $\begin{array}{l}50 \\
80\end{array}$ \\
\hline LGG_PA_PF & 279 & 0 & 279 & 0.0 & $\begin{array}{l}50 \\
80\end{array}$ \\
\hline LGG_SEGA & 29 & 0 & 29 & 0.0 & $\begin{array}{l}50 \\
80\end{array}$ \\
\hline LYMPHO & 39 & 0 & 39 & 0.0 & $\begin{array}{l}50 \\
80\end{array}$ \\
\hline
\end{tabular}




\begin{tabular}{|c|c|c|c|c|}
\hline MB_WNT & 125 & 0 & 125 & 0.0 \\
\hline MB_SHH_CHL_AD & 253 & 0 & 253 & 0.0 \\
\hline MB_SHH_INF & 142 & 0 & 142 & 0.0 \\
\hline MB_G3 & 257 & 0 & 257 & 0.0 \\
\hline MB_G4 & 513 & 0 & 513 & 0.0 \\
\hline MELCYT & 56 & 1 & 55 & 1.8 \\
\hline MELAN & 87 & 0 & 87 & 0.0 \\
\hline SCHW_MEL & 13 & 0 & 13 & 0.0 \\
\hline MNG & 1058 & 1 & 1057 & 0.1 \\
\hline PTPR_A & 11 & 0 & 11 & 0.0 \\
\hline PTPR_B & 32 & 0 & 32 & 0.0 \\
\hline PGG_nC & 24 & 0 & 24 & 0.0 \\
\hline PIN_T_PPT & 37 & 0 & 37 & 0.0 \\
\hline PIN_T_PB_A & 24 & 0 & 24 & 0.0 \\
\hline PIN_T_PB_B & 43 & 0 & 43 & 0.0 \\
\hline PITUI & 55 & 0 & 55 & 0.0 \\
\hline PITAD_ACTH & 48 & 0 & 48 & 0.0 \\
\hline PITAD_FSH_LH & 53 & 0 & 53 & 0.0 \\
\hline गITAD_STH_DNS_t & 15 & 0 & 15 & 0.0 \\
\hline गTAD_STH_DNS_E & 27 & 0 & 27 & 0.0 \\
\hline PITAD_STH_SPA & 28 & 0 & 28 & 0.0 \\
\hline PITAD_TSH & 25 & 0 & 25 & 0.0 \\
\hline PITAD_PRL & 17 & 1 & 16 & 5.9 \\
\hline PLASMA & 10 & 0 & 10 & 0.0 \\
\hline PLEX_AD & 36 & 0 & 36 & 0.0 \\
\hline
\end{tabular}




$\begin{array}{cccccl}\text { PLEX_PED_A } & 32 & 1 & 31 & 3.1 & 50 \\ \text { PLEX_PED_B } & 307 & 7 & 300 & 2.3 & 80 \\ \text { SCHW } & 155 & 1 & 154 & 0.6 & 80 \\ & & & & & 50 \\ \text { SFT_HMPC } & 37 & 0 & 37 & 0.0 & 50 \\ \text { SUBEPN_PF } & 54 & 1 & 53 & 1.9 & 80 \\ & & & & & 50 \\ \text { SUBEPN_SPINE } & 19 & 0 & 19 & 0.0 & 50 \\ & & & & & 80 \\ \text { SUBEPN_ST } & 25 & 0 & 25 & 0.0 & 50\end{array}$




\begin{tabular}{|c|c|c|}
\hline $7 p_{w t} 7 q_{w t}-10 p_{w t} 10 q_{w t}$ & $7 p_{w t}=7 q_{w t \_} 10 p_{w t \_} 10 q_{\text {loss }}$ & $7 p_{w t}=7 q_{w t} 10 p_{\text {loss } \_} 10 q_{w t}$ \\
\hline 90 & 6 & 5 \\
\hline 93 & 4 & 5 \\
\hline 71 & 1 & 3 \\
\hline 72 & 1 & 3 \\
\hline 57 & 1 & 0 \\
\hline 57 & 1 & 0 \\
\hline 44 & 2 & 0 \\
\hline 44 & 2 & 0 \\
\hline 39 & 10 & 0 \\
\hline 45 & 4 & 1 \\
\hline 96 & 3 & 3 \\
\hline 96 & 3 & 3 \\
\hline 460 & 4 & 2 \\
\hline 481 & 1 & 2 \\
\hline 416 & 22 & 2 \\
\hline 490 & 8 & 2 \\
\hline 133 & 79 & 4 \\
\hline 205 & 38 & 7 \\
\hline 70 & 7 & 14 \\
\hline 79 & 4 & 13 \\
\hline 76 & 0 & 3 \\
\hline 77 & 0 & 2 \\
\hline 98 & 2 & 0 \\
\hline 98 & 2 & 0 \\
\hline 57 & 0 & 0 \\
\hline 57 & 0 & 0 \\
\hline 30 & 0 & 0 \\
\hline 30 & 0 & 0 \\
\hline 14 & 1 & 0 \\
\hline 14 & 1 & 0 \\
\hline 17 & 0 & 0 \\
\hline 17 & 0 & 0 \\
\hline 27 & 0 & 1 \\
\hline 27 & 2 & 1 \\
\hline 36 & 0 & 1 \\
\hline 36 & 0 & 1 \\
\hline 40 & 1 & 2 \\
\hline 40 & 1 & 2 \\
\hline 18 & 0 & 0 \\
\hline 18 & 0 & 0 \\
\hline 180 & 40 & 3 \\
\hline 199 & 27 & 4 \\
\hline 76 & 0 & 6 \\
\hline 78 & 0 & 6 \\
\hline 128 & 1 & 2 \\
\hline 128 & 1 & 2 \\
\hline 15 & 0 & 0 \\
\hline 15 & 0 & 0 \\
\hline
\end{tabular}




\begin{tabular}{|c|c|}
\hline 23 & 0 \\
\hline 23 & 0 \\
\hline 317 & 10 \\
\hline 319 & 8 \\
\hline 6 & 0 \\
\hline 6 & 0 \\
\hline 28 & 0 \\
\hline 28 & 0 \\
\hline 2 & 0 \\
\hline 2 & 0 \\
\hline 2 & 0 \\
\hline 2 & 0 \\
\hline 36 & 21 \\
\hline 57 & 5 \\
\hline 11 & 6 \\
\hline 15 & 4 \\
\hline 4 & 4 \\
\hline 4 & 5 \\
\hline 6 & 3 \\
\hline 9 & 3 \\
\hline 13 & 4 \\
\hline 15 & 3 \\
\hline 39 & 7 \\
\hline 42 & 7 \\
\hline 68 & 9 \\
\hline 81 & 6 \\
\hline 56 & 0 \\
\hline 56 & 0 \\
\hline 32 & 0 \\
\hline 32 & 0 \\
\hline 87 & 0 \\
\hline 87 & 0 \\
\hline 15 & 0 \\
\hline 15 & 0 \\
\hline 120 & 0 \\
\hline 120 & 0 \\
\hline 53 & 3 \\
\hline 55 & 1 \\
\hline 24 & 0 \\
\hline 25 & 0 \\
\hline 84 & 0 \\
\hline 85 & 0 \\
\hline 106 & 0 \\
\hline 107 & 0 \\
\hline 244 & 1 \\
\hline 245 & 1 \\
\hline 29 & 0 \\
\hline 29 & 0 \\
\hline 27 & 0 \\
\hline 30 & 0 \\
\hline
\end{tabular}




\begin{tabular}{|c|c|c|}
\hline 103 & 3 & 3 \\
\hline 105 & 1 & 3 \\
\hline 184 & 39 & 1 \\
\hline 200 & 25 & 1 \\
\hline 106 & 9 & 2 \\
\hline 108 & 7 & 2 \\
\hline 76 & 21 & 1 \\
\hline 100 & 7 & 1 \\
\hline 248 & 13 & 6 \\
\hline 270 & 3 & 7 \\
\hline 53 & 2 & 0 \\
\hline 53 & 2 & 0 \\
\hline 27 & 4 & 5 \\
\hline 30 & 5 & 6 \\
\hline 8 & 0 & 0 \\
\hline 8 & 0 & 0 \\
\hline 828 & 44 & 18 \\
\hline 841 & 37 & 1. \\
\hline 1 & 0 & 0 \\
\hline 2 & 0 & 0 \\
\hline 1 & 0 & 0 \\
\hline 1 & 0 & 0 \\
\hline 24 & 0 & 0 \\
\hline 24 & 0 & 0 \\
\hline 29 & 0 & 1 \\
\hline 29 & 0 & 1 \\
\hline 19 & 1 & 0 \\
\hline 20 & 0 & 0 \\
\hline 16 & 1 & 0 \\
\hline 18 & 0 & 0 \\
\hline 50 & 1 & 0 \\
\hline 51 & 0 & 0 \\
\hline 32 & 0 & 0 \\
\hline 32 & 0 & 0 \\
\hline 51 & 1 & 0 \\
\hline 52 & 0 & 0 \\
\hline 3 & 0 & 0 \\
\hline 3 & 0 & 0 \\
\hline 21 & 3 & 0 \\
\hline 22 & 2 & 0 \\
\hline 28 & 0 & 0 \\
\hline 28 & 0 & 0 \\
\hline 9 & 1 & 0 \\
\hline 9 & 1 & 0 \\
\hline 1 & 1 & 0 \\
\hline 1 & 1 & C \\
\hline 6 & 0 & C \\
\hline 6 & 0 & C \\
\hline 1 & 0 & 0 \\
\hline 1 & 0 & \\
\hline
\end{tabular}




$\begin{array}{ccc}18 & 0 & 0 \\ 18 & 0 & 0 \\ 172 & 16 & 18 \\ 177 & 14 & 19 \\ 146 & 0 & 2 \\ 147 & 0 & 1 \\ 37 & 0 & 0 \\ 37 & 0 & 0 \\ 50 & 1 & 1 \\ 51 & 0 & 1 \\ 18 & 0 & 1 \\ 18 & 0 & 1 \\ 25 & 0 & 0 \\ 25 & 0 & 0\end{array}$




\begin{tabular}{|c|c|c|}
\hline $7 p_{\text {wt_ }} 7 q_{\text {wt_ }} 10 p_{\text {loss_ }} 10 q_{\text {loss }}$ & $7 p_{w t \_} 7 q_{\text {gain_}} 10 p_{w t \_} 10 q_{w t}$ & $7 p_{w t \_} 7 q_{\text {gain_}} 10 p_{w t \_} 10 q_{\text {loss }}$ \\
\hline 32 & 2 & 0 \\
\hline 31 & 3 & 0 \\
\hline 3 & 1 & 0 \\
\hline 4 & 1 & 0 \\
\hline 1 & 0 & 0 \\
\hline 1 & 0 & 0 \\
\hline 10 & 0 & 0 \\
\hline 10 & 0 & 0 \\
\hline 2 & 0 & 0 \\
\hline 1 & 0 & 0 \\
\hline 11 & 0 & 0 \\
\hline 11 & 0 & 0 \\
\hline 12 & 23 & 0 \\
\hline 12 & 7 & 0 \\
\hline 0 & 131 & 5 \\
\hline 0 & 80 & 1 \\
\hline 32 & 33 & 16 \\
\hline 31 & 20 & 5 \\
\hline 6 & 5 & 0 \\
\hline 6 & 3 & 0 \\
\hline 1 & 0 & 0 \\
\hline 1 & 0 & 0 \\
\hline 5 & 0 & 0 \\
\hline 5 & 0 & 0 \\
\hline 0 & 0 & 0 \\
\hline 0 & 0 & 0 \\
\hline 0 & 0 & 0 \\
\hline 0 & 0 & 0 \\
\hline 1 & 0 & 0 \\
\hline 1 & 0 & 0 \\
\hline 0 & 0 & 0 \\
\hline 0 & 0 & 0 \\
\hline 19 & 1 & 1 \\
\hline 18 & 1 & 0 \\
\hline 0 & 0 & 0 \\
\hline 0 & 0 & 0 \\
\hline 3 & 0 & 0 \\
\hline 3 & 0 & 0 \\
\hline 2 & 4 & 0 \\
\hline 2 & 4 & 0 \\
\hline 26 & 6 & 3 \\
\hline 25 & 6 & 3 \\
\hline 7 & 0 & 0 \\
\hline 7 & 0 & 0 \\
\hline 16 & 0 & 0 \\
\hline 16 & 0 & 0 \\
\hline 0 & 0 & 0 \\
\hline 0 & 0 & 0 \\
\hline
\end{tabular}




\begin{tabular}{|c|c|c|}
\hline 17 & 0 & 0 \\
\hline 17 & 0 & 0 \\
\hline 7 & 1 & 0 \\
\hline 7 & 1 & 0 \\
\hline 18 & 0 & c \\
\hline 18 & 0 & 0 \\
\hline 5 & 0 & c \\
\hline 5 & 0 & c \\
\hline 9 & 0 & 0 \\
\hline 9 & 0 & 0 \\
\hline 16 & 0 & 0 \\
\hline 16 & 0 & 0 \\
\hline 4 & 5 & 1 \\
\hline 2 & 5 & c \\
\hline 9 & 3 & 2 \\
\hline 9 & 4 & c \\
\hline 39 & 0 & 0 \\
\hline 42 & 2 & 0 \\
\hline 82 & 0 & 2 \\
\hline 98 & 1 & 2 \\
\hline 6 & 0 & 0 \\
\hline 5 & 0 & c \\
\hline 108 & 6 & 3 \\
\hline 114 & 7 & 2 \\
\hline 19 & 5 & c \\
\hline 17 & 3 & 0 \\
\hline 1 & 0 & 0 \\
\hline 1 & 0 & 0 \\
\hline 2 & 0 & c \\
\hline 2 & 0 & 0 \\
\hline 0 & 0 & C \\
\hline 0 & 0 & c \\
\hline 1 & 0 & 0 \\
\hline 1 & 0 & c \\
\hline 3 & 1 & 0 \\
\hline 3 & 1 & C \\
\hline 0 & 1 & 0 \\
\hline 0 & 1 & c \\
\hline 0 & 1 & 0 \\
\hline 0 & 0 & 0 \\
\hline 2 & 1 & c \\
\hline 2 & 1 & 0 \\
\hline 0 & 0 & c \\
\hline 0 & 0 & c \\
\hline 1 & 1 & 0 \\
\hline 1 & 2 & 0 \\
\hline 0 & 0 & 0 \\
\hline 0 & 0 & c \\
\hline 2 & 3 & C \\
\hline 1 & 2 & 0 \\
\hline
\end{tabular}




\begin{tabular}{|c|c|c|}
\hline 9 & 1 & 0 \\
\hline 9 & 1 & 0 \\
\hline 14 & 4 & 1 \\
\hline 12 & 5 & 1 \\
\hline 13 & 0 & 0 \\
\hline 13 & 0 & 0 \\
\hline 38 & 10 & 13 \\
\hline 38 & 14 & 2 \\
\hline 15 & 24 & 3 \\
\hline 16 & 17 & 0 \\
\hline 0 & 0 & 0 \\
\hline 0 & 0 & 0 \\
\hline 12 & 2 & 2 \\
\hline 13 & 2 & 2 \\
\hline 1 & 0 & 0 \\
\hline 1 & 0 & 0 \\
\hline 103 & 20 & 3 \\
\hline 102 & 18 & 2 \\
\hline 6 & 0 & 0 \\
\hline 6 & 0 & 0 \\
\hline 30 & 0 & 0 \\
\hline 30 & 0 & 0 \\
\hline 0 & 0 & 0 \\
\hline 0 & 0 & 0 \\
\hline 4 & 0 & 0 \\
\hline 4 & 0 & 0 \\
\hline 1 & 0 & 0 \\
\hline 1 & 0 & 0 \\
\hline 3 & 2 & 1 \\
\hline 4 & 1 & 1 \\
\hline 1 & 1 & 0 \\
\hline 1 & 1 & 0 \\
\hline 10 & 0 & 0 \\
\hline 10 & 0 & 0 \\
\hline 0 & 0 & 0 \\
\hline 0 & 0 & 0 \\
\hline 1 & 0 & 0 \\
\hline 1 & 0 & 0 \\
\hline 0 & 0 & 0 \\
\hline 0 & 0 & 0 \\
\hline 0 & 0 & 0 \\
\hline 0 & 0 & c \\
\hline 4 & 0 & 0 \\
\hline 4 & 0 & C \\
\hline 0 & 0 & 0 \\
\hline 0 & 0 & c \\
\hline 1 & 0 & c \\
\hline 1 & 0 & C \\
\hline 2 & 1 & c \\
\hline 2 & 1 & \\
\hline
\end{tabular}




$\begin{array}{lll}2 & 0 & 0 \\ 2 & 0 & 0 \\ 44 & 2 & 0 \\ 44 & 2 & 0 \\ 0 & 0 & 0 \\ 0 & 0 & 0 \\ 0 & 0 & 0 \\ 0 & 0 & 0 \\ 0 & 0 & 0 \\ 0 & 0 & 0 \\ 0 & 0 & 0 \\ 0 & 0 & 0 \\ 0 & 0 & 0 \\ 0 & 0 & 0\end{array}$




\begin{tabular}{|c|c|c|}
\hline $7 p_{\text {wt_ }} 7 q_{\text {gain_} \_} 10 p_{\text {loss_ }} 10 q_{w t}$ & $7 p_{\text {wt_- }} 7 q_{\text {gain__ }} 10 p_{\text {loss_ }} 10 q_{\text {loss }}$ & $7 p_{\text {gain_}} 7 q_{w t \_} 10 p_{w t \_} 10 q_{w t}$ \\
\hline 1 & 3 & 3 \\
\hline 0 & 3 & 4 \\
\hline 0 & 0 & 0 \\
\hline 0 & 0 & 0 \\
\hline 0 & 0 & 0 \\
\hline 0 & 0 & 0 \\
\hline 0 & 0 & 0 \\
\hline 0 & 0 & 0 \\
\hline 0 & 0 & 0 \\
\hline 0 & 0 & 0 \\
\hline 0 & 0 & 2 \\
\hline 0 & 0 & 2 \\
\hline 1 & 0 & 2 \\
\hline 1 & 0 & 1 \\
\hline 1 & 0 & 8 \\
\hline 1 & 0 & 6 \\
\hline 1 & 5 & 5 \\
\hline 0 & 2 & 5 \\
\hline 0 & 1 & 2 \\
\hline 0 & 0 & 2 \\
\hline 0 & 0 & 0 \\
\hline 0 & 0 & 0 \\
\hline 0 & 0 & 0 \\
\hline 0 & 0 & 0 \\
\hline 0 & 0 & 0 \\
\hline 0 & 0 & 0 \\
\hline 0 & 0 & 0 \\
\hline 0 & 0 & 0 \\
\hline 0 & 0 & 0 \\
\hline 0 & 0 & 0 \\
\hline 0 & 0 & 0 \\
\hline 0 & 0 & 0 \\
\hline 1 & 0 & 1 \\
\hline 1 & 0 & 2 \\
\hline 0 & 0 & 0 \\
\hline 0 & 0 & 1 \\
\hline 0 & 0 & 1 \\
\hline 0 & 0 & 2 \\
\hline 0 & 0 & 0 \\
\hline 0 & 0 & 0 \\
\hline 0 & 0 & 10 \\
\hline 0 & 0 & 8 \\
\hline 0 & 0 & 3 \\
\hline 0 & 0 & 2 \\
\hline 0 & 0 & 0 \\
\hline 0 & 0 & 0 \\
\hline 0 & 0 & 0 \\
\hline 0 & 0 & 0 \\
\hline
\end{tabular}




\begin{tabular}{|c|c|}
\hline 0 & 0 \\
\hline 0 & 0 \\
\hline 0 & 0 \\
\hline 0 & 0 \\
\hline 0 & 0 \\
\hline 0 & 0 \\
\hline 0 & 0 \\
\hline 0 & 0 \\
\hline 0 & 0 \\
\hline 0 & 0 \\
\hline 0 & 0 \\
\hline 0 & 0 \\
\hline 0 & 3 \\
\hline 0 & 3 \\
\hline 1 & 0 \\
\hline 0 & 0 \\
\hline 0 & 8 \\
\hline 0 & 9 \\
\hline 1 & 35 \\
\hline 0 & 53 \\
\hline 1 & 0 \\
\hline 0 & 0 \\
\hline 3 & 24 \\
\hline 1 & 32 \\
\hline 0 & 0 \\
\hline 0 & 0 \\
\hline 0 & 0 \\
\hline 0 & 0 \\
\hline 0 & 0 \\
\hline 0 & 0 \\
\hline 0 & 0 \\
\hline 0 & 0 \\
\hline 0 & 0 \\
\hline 0 & 0 \\
\hline 0 & 0 \\
\hline 0 & 0 \\
\hline 0 & 0 \\
\hline 0 & 0 \\
\hline 0 & 0 \\
\hline 0 & 0 \\
\hline 0 & 0 \\
\hline 0 & 0 \\
\hline 0 & 0 \\
\hline 0 & 0 \\
\hline 0 & 0 \\
\hline 0 & 0 \\
\hline 0 & 0 \\
\hline 0 & 0 \\
\hline 0 & 0 \\
\hline 0 & 0 \\
\hline
\end{tabular}




\begin{tabular}{|c|c|}
\hline 0 & 0 \\
\hline 0 & 0 \\
\hline 0 & 1 \\
\hline 0 & 1 \\
\hline 0 & 0 \\
\hline 0 & 0 \\
\hline 1 & 3 \\
\hline 1 & 4 \\
\hline 1 & 3 \\
\hline 0 & 2 \\
\hline 0 & 0 \\
\hline 0 & 0 \\
\hline 3 & 4 \\
\hline 1 & 2 \\
\hline 0 & 1 \\
\hline 0 & 1 \\
\hline 0 & 3 \\
\hline 0 & 3 \\
\hline 0 & 0 \\
\hline 0 & 0 \\
\hline 0 & 0 \\
\hline 0 & 0 \\
\hline 0 & 0 \\
\hline 0 & 0 \\
\hline 0 & 0 \\
\hline 0 & 0 \\
\hline 0 & 0 \\
\hline 0 & 0 \\
\hline 0 & 1 \\
\hline 0 & 0 \\
\hline 0 & 0 \\
\hline 0 & 0 \\
\hline 0 & 0 \\
\hline 0 & 0 \\
\hline 0 & 0 \\
\hline 0 & 0 \\
\hline 0 & 0 \\
\hline 0 & 0 \\
\hline 0 & 1 \\
\hline 0 & 1 \\
\hline 0 & 0 \\
\hline 0 & 0 \\
\hline 0 & 0 \\
\hline 0 & 0 \\
\hline 0 & 0 \\
\hline 0 & 0 \\
\hline 0 & 0 \\
\hline 0 & 0 \\
\hline 0 & 0 \\
\hline 0 & 0 \\
\hline
\end{tabular}




$\begin{array}{ll}0 & 0 \\ 0 & 0 \\ 1 & 2 \\ 0 & 2 \\ 0 & 0 \\ 0 & 0 \\ 0 & 0 \\ 0 & 0 \\ 0 & 0 \\ 0 & 0 \\ 0 & 0 \\ 0 & 0 \\ 0 & 0 \\ 0 & 0\end{array}$




\begin{tabular}{|c|c|c|}
\hline $7 p_{\text {gain_}} 7 q_{\text {wt_ }} 10 p_{\text {wt_ }} 10 q_{\text {loss }}$ & $7 p_{\text {gain_}} 7 q_{\text {wt_ }} 10 p_{\text {loss_- }} 10 q_{w t}$ & $7 p_{\text {gain_ }} 7 q_{\text {wt_ }} 10 p_{\text {loss_}} 10 q_{\text {loss }}$ \\
\hline 0 & 0 & 1 \\
\hline 0 & 0 & 1 \\
\hline 0 & 1 & 1 \\
\hline 0 & 1 & 0 \\
\hline 0 & 0 & 0 \\
\hline 0 & 0 & 0 \\
\hline 0 & 0 & 0 \\
\hline 0 & 0 & 0 \\
\hline 0 & 0 & 0 \\
\hline 0 & 0 & 0 \\
\hline 0 & 0 & 0 \\
\hline 0 & 0 & 0 \\
\hline 0 & 0 & 0 \\
\hline 0 & 0 & 0 \\
\hline 0 & 0 & 0 \\
\hline 0 & 0 & 0 \\
\hline 6 & 0 & 0 \\
\hline 3 & 0 & 0 \\
\hline 0 & 1 & 0 \\
\hline 0 & 0 & 0 \\
\hline 0 & 0 & 0 \\
\hline 0 & 0 & 0 \\
\hline 0 & 0 & 0 \\
\hline 0 & 0 & 0 \\
\hline 0 & 0 & 0 \\
\hline 0 & 0 & 0 \\
\hline 0 & 0 & 0 \\
\hline 0 & 0 & 0 \\
\hline 0 & 0 & 0 \\
\hline 0 & 0 & 0 \\
\hline 0 & 0 & 0 \\
\hline 0 & 0 & 0 \\
\hline 1 & 0 & 0 \\
\hline 1 & 0 & 0 \\
\hline 0 & 0 & 0 \\
\hline 0 & 0 & 0 \\
\hline 1 & 0 & 0 \\
\hline 1 & 0 & 0 \\
\hline 0 & 0 & 0 \\
\hline 0 & 0 & 0 \\
\hline 2 & 2 & 0 \\
\hline 1 & 1 & 0 \\
\hline 0 & 2 & 0 \\
\hline 0 & 2 & 0 \\
\hline 0 & 0 & 0 \\
\hline 0 & 0 & 0 \\
\hline 0 & 0 & 0 \\
\hline 0 & 0 & 0 \\
\hline
\end{tabular}




\begin{tabular}{|c|c|c|}
\hline 0 & 0 & 1 \\
\hline 0 & 0 & 1 \\
\hline 0 & 0 & 0 \\
\hline 0 & 0 & 0 \\
\hline 0 & 0 & 0 \\
\hline 0 & 0 & 0 \\
\hline 0 & 0 & 0 \\
\hline 0 & 0 & 0 \\
\hline 0 & 0 & 1 \\
\hline 0 & 0 & 1 \\
\hline 0 & 0 & 1 \\
\hline 0 & 0 & 1 \\
\hline 2 & 0 & 1 \\
\hline 2 & 0 & 3 \\
\hline 0 & 0 & 3 \\
\hline 0 & 0 & 3 \\
\hline 2 & 1 & 12 \\
\hline 3 & 0 & 18 \\
\hline 3 & 0 & 30 \\
\hline 1 & 0 & 35 \\
\hline 0 & 0 & 1 \\
\hline 0 & 0 & 1 \\
\hline 1 & 1 & 25 \\
\hline 1 & 1 & 32 \\
\hline 3 & 1 & 2 \\
\hline 0 & 0 & 2 \\
\hline 0 & 0 & 0 \\
\hline 0 & 0 & 0 \\
\hline 0 & 0 & 0 \\
\hline 0 & 0 & 0 \\
\hline 0 & 0 & 0 \\
\hline 0 & 0 & 0 \\
\hline 0 & 0 & 0 \\
\hline 0 & 0 & 0 \\
\hline 0 & 0 & 1 \\
\hline 0 & 0 & 1 \\
\hline 0 & 0 & 0 \\
\hline 0 & 0 & 0 \\
\hline 0 & 0 & 0 \\
\hline 0 & 0 & 0 \\
\hline 0 & 0 & 0 \\
\hline 0 & 0 & 0 \\
\hline 0 & 0 & 0 \\
\hline 0 & 0 & 0 \\
\hline 0 & 0 & 0 \\
\hline 0 & 0 & 0 \\
\hline 0 & 0 & 0 \\
\hline 0 & 0 & 0 \\
\hline 0 & 0 & 0 \\
\hline 0 & 0 & \\
\hline
\end{tabular}




\begin{tabular}{|c|c|c|}
\hline 0 & 0 & 0 \\
\hline 0 & 0 & 0 \\
\hline 0 & 0 & 0 \\
\hline 0 & 0 & 0 \\
\hline 0 & 0 & 0 \\
\hline 0 & 0 & 0 \\
\hline 2 & 0 & 0 \\
\hline 0 & 0 & 0 \\
\hline 0 & 1 & 1 \\
\hline 0 & 1 & 1 \\
\hline 0 & 0 & 0 \\
\hline 0 & 0 & 0 \\
\hline 1 & 1 & 0 \\
\hline 1 & 1 & 0 \\
\hline 0 & 0 & 0 \\
\hline 0 & 0 & 0 \\
\hline 0 & 0 & 2 \\
\hline 0 & 0 & 2 \\
\hline 0 & 0 & 0 \\
\hline 0 & 0 & 0 \\
\hline 0 & 0 & 0 \\
\hline 0 & 0 & 0 \\
\hline 0 & 0 & 0 \\
\hline 0 & 0 & 0 \\
\hline 0 & 0 & 0 \\
\hline 0 & 0 & 0 \\
\hline 0 & 0 & 0 \\
\hline 0 & 0 & 0 \\
\hline 0 & 0 & 0 \\
\hline 0 & 0 & 0 \\
\hline 0 & 0 & 0 \\
\hline 0 & 0 & 0 \\
\hline 0 & 0 & 1 \\
\hline 0 & 0 & 1 \\
\hline 0 & 0 & 0 \\
\hline 0 & 0 & 0 \\
\hline 0 & 0 & 0 \\
\hline 0 & 0 & 0 \\
\hline 0 & 0 & 0 \\
\hline 0 & 0 & 0 \\
\hline 0 & 0 & 0 \\
\hline 0 & 0 & 0 \\
\hline 0 & 0 & 0 \\
\hline 0 & 0 & 0 \\
\hline 0 & 0 & 0 \\
\hline 0 & 0 & 0 \\
\hline 0 & 0 & 0 \\
\hline 0 & 0 & 0 \\
\hline 1 & 0 & 0 \\
\hline 1 & 0 & 0 \\
\hline
\end{tabular}




$\begin{array}{lll}0 & 0 & 0 \\ 0 & 0 & 0 \\ 5 & 0 & 7 \\ 4 & 0 & 4 \\ 0 & 0 & 0 \\ 0 & 0 & 0 \\ 0 & 0 & 0 \\ 0 & 0 & 0 \\ 0 & 0 & 0 \\ 0 & 0 & 0 \\ 0 & 0 & 0 \\ 0 & 0 & 0 \\ 0 & 0 & 0 \\ 0 & 0 & 0\end{array}$




\begin{tabular}{|c|c|c|}
\hline $7 p_{\text {gain_}} 7 q_{\text {gain__ }} 10 p_{w t \_} 10 q_{w t}$ & $7 p_{\text {gain_}} 7 q_{\text {gain_}} 10 p_{\text {wt_ }} 10 q_{\text {loss }}$ & $7 p_{\text {gain_}} 7 q_{\text {gain_}} 10 p_{\text {loss }} 10 q_{w t}$ \\
\hline 38 & 0 & 2 \\
\hline 37 & 0 & 3 \\
\hline 2 & 0 & 1 \\
\hline 2 & 0 & 0 \\
\hline 2 & 0 & 1 \\
\hline 2 & 0 & 1 \\
\hline 1 & 0 & 0 \\
\hline 1 & 0 & 0 \\
\hline 4 & 1 & 0 \\
\hline 5 & 0 & 0 \\
\hline 5 & 0 & 1 \\
\hline 5 & 0 & 1 \\
\hline 22 & 1 & 0 \\
\hline 21 & 1 & 0 \\
\hline 53 & 2 & 0 \\
\hline 52 & 0 & 0 \\
\hline 25 & 10 & 1 \\
\hline 30 & 3 & 1 \\
\hline 5 & 0 & 0 \\
\hline 4 & 0 & 0 \\
\hline 1 & 0 & 0 \\
\hline 1 & 0 & 0 \\
\hline 2 & 0 & 0 \\
\hline 2 & 0 & 0 \\
\hline 4 & 0 & 0 \\
\hline 4 & 0 & 0 \\
\hline 0 & 0 & 0 \\
\hline 0 & 0 & 0 \\
\hline 0 & 0 & 0 \\
\hline 0 & 0 & 0 \\
\hline 0 & 0 & 0 \\
\hline 0 & 0 & 0 \\
\hline 10 & 1 & 0 \\
\hline 10 & 0 & 0 \\
\hline 3 & 0 & 1 \\
\hline 2 & 0 & 1 \\
\hline 1 & 0 & 0 \\
\hline 0 & 0 & 0 \\
\hline 5 & 0 & 0 \\
\hline 5 & 0 & 0 \\
\hline 16 & 1 & 0 \\
\hline 15 & 0 & 0 \\
\hline 11 & 1 & 0 \\
\hline 10 & 1 & 0 \\
\hline 10 & 0 & 2 \\
\hline 11 & 0 & 1 \\
\hline 0 & 0 & 0 \\
\hline 0 & 0 & 0 \\
\hline
\end{tabular}




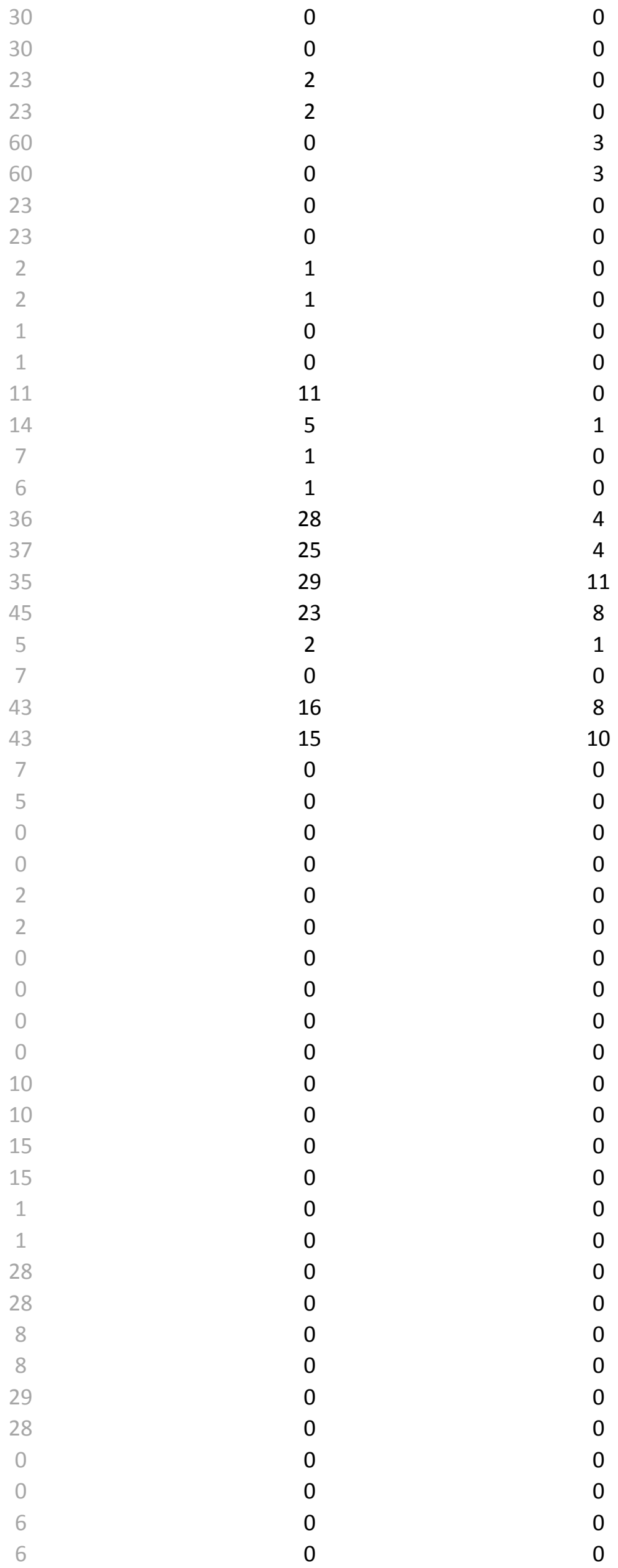




\begin{tabular}{|c|c|c|}
\hline 5 & 0 & 0 \\
\hline 5 & 0 & 0 \\
\hline 7 & 1 & 0 \\
\hline 7 & 0 & 0 \\
\hline 10 & 1 & 0 \\
\hline 11 & 0 & 0 \\
\hline 60 & 10 & 3 \\
\hline 66 & 4 & 2 \\
\hline 127 & 18 & 6 \\
\hline 134 & 8 & 6 \\
\hline 1 & 0 & 0 \\
\hline 1 & 0 & 0 \\
\hline 13 & 4 & 2 \\
\hline 13 & 3 & 1 \\
\hline 3 & 0 & 0 \\
\hline 3 & 0 & 0 \\
\hline 33 & 1 & 0 \\
\hline 33 & 1 & 0 \\
\hline 3 & 0 & 0 \\
\hline 2 & 0 & 0 \\
\hline 1 & 0 & 0 \\
\hline 1 & 0 & 0 \\
\hline 0 & 0 & 0 \\
\hline 0 & 0 & 0 \\
\hline 2 & 0 & 0 \\
\hline 2 & 0 & 0 \\
\hline 2 & 1 & 0 \\
\hline 2 & 1 & 0 \\
\hline 13 & 1 & 0 \\
\hline 14 & 0 & 0 \\
\hline 2 & 0 & 0 \\
\hline 2 & 0 & 0 \\
\hline 4 & 0 & 0 \\
\hline 4 & 0 & 0 \\
\hline 1 & 0 & 0 \\
\hline 1 & 0 & 0 \\
\hline 9 & 0 & 0 \\
\hline 9 & 0 & 0 \\
\hline 1 & 0 & 0 \\
\hline 1 & 0 & 0 \\
\hline 0 & 0 & 0 \\
\hline 0 & 0 & 0 \\
\hline 9 & 0 & 0 \\
\hline 9 & 0 & 0 \\
\hline 14 & 0 & 1 \\
\hline 15 & 0 & 0 \\
\hline 3 & 0 & 0 \\
\hline 3 & 0 & 0 \\
\hline 21 & 0 & 0 \\
\hline 21 & 0 & \\
\hline
\end{tabular}




$\begin{array}{lll}7 & 0 & 0 \\ 7 & 0 & 0 \\ 23 & 1 & 2 \\ 24 & 1 & 1 \\ 7 & 0 & 0 \\ 7 & 0 & 0 \\ 0 & 0 & 0 \\ 0 & 0 & 0 \\ 2 & 0 & 0 \\ 2 & 0 & 0 \\ 0 & 0 & 0 \\ 0 & 0 & 0 \\ 0 & 0 & 0 \\ 0 & 0 & 0\end{array}$


$7 p_{\text {gain_}} 7 q_{\text {gain__10 }} 10 p_{\text {loss_ }} 10 q_{\text {loss }}$

16

15

1

1

0

0

1

1

0

0

0

1

1

0

1

1

1

1

1

0

0

2

2

0

0

0

0

0

0

0

18

18

0

0

0

0

0

0

3

3

0

0

2

2

0

0 


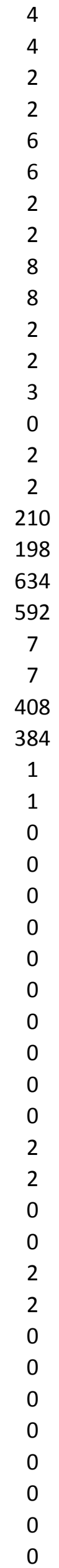




1
1
0
0
0
0
16
15
40
40
0
0
3
3
0
0
0
10
10
1
0
1
0
1
0
0
0
0
0
0
0
0
0
0
0
0
0
0
0
0
0
0
0
0
0
0
0
0
0
0
0
0
0
0
0
0
0
0
0
0
0
0
0
0
0
0


5
5
8
8
0
0
0
0
0
0
0
0
0
0 